\title{
¿Argovis: A Web Application for Fast Delivery, Visualization, and Analysis of Argo Data
}

\author{
TYLER TUCKER \\ Department of Atmospheric and Oceanic Sciences, University of Colorado Boulder, Boulder, Colorado, and \\ Department of Mathematics and Statistics, San Diego State University, San Diego, and Scripps Institution of Oceanography, \\ University of California, San Diego, La Jolla, California \\ DonAta Giglio \\ Department of Atmospheric and Oceanic Sciences, University of Colorado Boulder, Boulder, Colorado \\ MEGAN SCANDERBEG \\ Scripps Institution of Oceanography, University of California, San Diego, La Jolla, California \\ SAMUEL S. P. SHEN \\ Department of Mathematics and Statistics, San Diego State University, San Diego, California
}

(Manuscript received 18 March 2019, in final form 3 January 2020)

\begin{abstract}
Since the mid-2000s, the Argo oceanographic observational network has provided near-real-time fourdimensional data for the global ocean for the first time in history. Internet (i.e., the "web") applications that handle the more than two million Argo profiles of ocean temperature, salinity, and pressure are an active area of development. This paper introduces a new and efficient interactive Argo data visualization and delivery web application named Argovis that is built on a classic three-tier design consisting of a front end, back end, and database. Together these components allow users to navigate 4D data on a world map of Argo floats, with the option to select a custom region, depth range, and time period. Argovis's back end sends data to users in a simple format, and the front end quickly renders web-quality figures. More advanced applications query Argovis from other programming environments, such as Python, R, and MATLAB. Our Argovis architecture allows expert data users to build their own functionality for specific applications, such as the creation of spatially gridded data for a given time and advanced time-frequency analysis for a space-time selection. Argovis is aimed to both scientists and the public, with tutorials and examples available on the website, describing how to use the Argovis data delivery system-for example, how to plot profiles in a region over time or to monitor profile metadata.
\end{abstract}

\section{Introduction}

For the first time in history, the Argo network of profiling floats provides real-time data of temperature $T$, salinity $S$, and pressure $P$ for the global ocean to a depth of 2000 dbar, with Deep Argo floats going down to 6000-dbar depth. Argo floats have been deployed since the early 2000s and reached the expected spatial distribution in 2007 (Roemmich et al. 2009). Nearly

¿ Denotes content that is immediately available upon publication as open access.

Corresponding author: Tyler Tucker, tyler.tucker@colorado.edu
4000 floats are currently operating in the global ocean and provide a profile every 10 days, that is, measurements from a vertical column of the ocean as a single float ascends to the surface. The four-dimensional (4D) space-time Argo data have many scientific and technological advantages, two of which are 1) unprecedented spatial and temporal resolution over the global ocean, and 2) no seasonal bias (Roemmich et al. 2009).

More than two million $T / S / P$ profiles have been collected through the Argo Program. Datasets as complex and large as Argo raise data delivery and visualization challenges. Users need to extract relevant subsets without necessarily having the expertise and familiarity with data formatting practices or programming knowledge. 
TABLE 1. List of abbreviations along with context of how they apply to this paper.

\begin{tabular}{|c|c|c|}
\hline Abbreviation & Definition & Explanation \\
\hline ASCII & $\begin{array}{l}\text { American Standard Code for } \\
\text { Information Interchange }\end{array}$ & A file that contains unformatted text is called an ASCII or text file \\
\hline API & $\begin{array}{l}\text { Application programming } \\
\text { interface }\end{array}$ & $\begin{array}{l}\text { A set of methods of communication among various computers; in the context } \\
\text { of this paper, it allows software programs to request data from Argovis }\end{array}$ \\
\hline BGC & Biogeochemical & $\begin{array}{l}\text { Argo floats that are equipped with additional sensors that measure BGC } \\
\text { parameters, such as dissolved oxygen }\end{array}$ \\
\hline CPU & Central processing unit & $\begin{array}{l}\text { A microprocessor that is often described as the brain of a computer; it } \\
\text { specializes in sequential computing }\end{array}$ \\
\hline CSS & Cascading Style Sheets & $\begin{array}{l}\text { A language used for describing the presentation of elements on an HTML } \\
\text { document }\end{array}$ \\
\hline CSV & Comma-separated value & $\begin{array}{l}\text { A file type that stores tabular data in plain text, delimited by a separator } \\
\text { (such as a comma) }\end{array}$ \\
\hline DAC & Data assembly center & National DACs gather and perform QC on floats owned by that nation \\
\hline dbar & Decibar & Unit of pressure; $1 \mathrm{dbar}$ is approximately equal to $1-\mathrm{m}$ depth of seawater \\
\hline FTP & File transfer protocol & A standard network protocol used to transfer files on a computer network \\
\hline GDACs & Global DAC & GDACs store and maintain Argo data sent by DACS \\
\hline GPS & Global positioning system & $\begin{array}{l}\text { A satellite-based radionavigation system that some Argo floats use to } \\
\text { communicate with their respective DACs }\end{array}$ \\
\hline GPU & Graphics processing unit & $\begin{array}{l}\text { A microprocessor that specializes in parallel computing, such as rendering } \\
\text { graphics }\end{array}$ \\
\hline HTML & Hypertext Markup Language & $\begin{array}{l}\text { A language for documents designed to be displayed in a web browser; often } \\
\text { used with CSS and JavaScript }\end{array}$ \\
\hline HTTP & Hypertext Transfer Protocol & $\begin{array}{l}\text { "HTTP is the underlying protocol used by the [web; it] defines how messages } \\
\text { are formatted and transmitted, and what actions web servers and browsers } \\
\text { should take in response to various commands" (V. Beal; https://www. } \\
\text { webopedia.com/TERM/H/HTTP.html) }\end{array}$ \\
\hline JSON & JavaScript Object Notation & $\begin{array}{l}\text { An open-standard file format text to transmit data objects consisting of } \\
\text { key-value pairs }\end{array}$ \\
\hline KML & Keyhole Markup Language & $\begin{array}{l}\text { A file type for expressing geographic annotation and visualization within } \\
\text { Google Earth }\end{array}$ \\
\hline NetCDF & Network Common Data Form & A type of file designed to house array-oriented scientific data \\
\hline QC & Quality control & The process of assigning a ranking at which processed Argo data are rated \\
\hline RESTful & Representational State Transfer & $\begin{array}{l}\text { A software architectural style that defines a set of constraints to be used for } \\
\text { creating web services; Argovis is RESTful because 1) it uses a base URL, } \\
\text { 2) it has an API interface that accepts "GET" requests, and 3) it sends } \\
\text { data as JSON }\end{array}$ \\
\hline SQL & Structured Query Language & $\begin{array}{l}\text { A language used to create, maintain, and retrieve relational databases such } \\
\text { as MySQL or Postgres }\end{array}$ \\
\hline$T / S / P$ & Temperature, salinity, and pressure & Core variables measured by each Argo float \\
\hline URL & Uniform resource locator & $\begin{array}{l}\text { An address identifying where documents or web pages can be found on the } \\
\text { Internet }\end{array}$ \\
\hline WMO & World Meteorological Organization & Argo floats are designated by a unique WMO number \\
\hline
\end{tabular}

The International Argo Program, plus the national programs that contribute to it, makes Argo data available online to anyone, with global data assembly centers (GDACs; for convenience, the acronyms and abbreviations used in this paper are also defined in Table 1) storing the data online. Users download either single profiles or all of the profiles taken from a single float over its entire history of deployment. With little option of space-time or metadata selection, this method is inefficient for many scientific and/or technical applications and discourages use by laypeople and policy makers.

An ongoing effort has been made within the Argo community to improve visualization and delivery of
Argo data and their derivative products to scientists, policy makers, and the general public. Some visualization tools are currently available online. For example, the Joint Technical Commission for Oceanography and Marine Meteorology In Situ Observations Programme Support Centre (JCOMMOPS) includes an interactive map (at http://argo.jcommops.org) that prompts users to query floats by program (Core Argo, Biogeochemical Argo, Deep Argo, etc.), nation, float type, last position, transmission type, and so on (JCOMMOPS 2019). The most recent profile of the selected floats appears on the interactive map with the option to show all positions of profiles within a size limit. The Argo-France group offers a 
product (at http://map.argo-france.fr/) that shows profile locations for the past 7 days with the option to click on individual profiles and see a plot of the data and previous locations for that float (Argo France 2019). Also, the EuroArgo group offers a similar Argo visualization application (at https://fleetmonitoring.euro-argo.eu/dashboard).

There are data delivery tools (for data other than Argo) whose design the Argo Program can borrow for their own application. The Web-Based Reanalyses Intercomparison Tools (WRIT) (at https://www.esrl. noaa.gov/psd/data/writ/) (Smith et al. 2014) allow users to create customized plots and retrieve climate data from these plots. WRIT addresses how to store and distribute large climate datasets, and helps to overcome data selection and delivery for scientists. The complex tabular WRIT interface is for scientists and professionals and not for the general public or, for example, high-school students.

A similar application is owned by NASA GES DISC. Giovanni (at https://giovanni.gsfc.nasa.gov/giovanni/), uses a dedicated server to create images, Keyhole Markup Language (KML) files used by Google Maps, and Network Common Data Form (netCDF) files. Giovanni depends on a server to handle WorldWide Web (hereinafter the "web") traffic, which requires hardware and maintenance. Alternatively, using the user's browser to generate plots and maps significantly reduces computational loads, thereby reducing the resources needed to host a data delivery web application.

The Four-Dimensional Visual Delivery (4DVD) technology (at http://4dvd.sdsu.edu/) (Pierret and Shen 2017) is one such tool: it combines data visualization and delivery while also having the user's computer create customized charts and maps. 4DVD displays gridded data and time series on an interactive globe for Global Precipitation Climatology Project (GPCP) data and NOAA CIRES reanalysis fields. Users make selections on a menu such as depth and date, to display data dynamically. Visualization elements such as charts and maps are rendered using the user's central processing unit (CPU) and graphics processing unit (GPU). This allows the webserver to focus on sending data quickly, requiring little computation power and ultimately improving its performance (Pierret and Shen 2017).

Following the ideas of the 4DVD technology, we created Argovis (at https://argovis.colorado.edu), a modern and efficient web application that combines web browser, webserver, and database technologies to navigate the Argo dataset in space and time. This web application provides two services: 1) visualizing Argo data and its gridded products with charts and maps and 2) delivering Argo data to the user. Through basic web browser navigation, Argovis enables a wide variety of users to use a world map of Argo floats, a depth range, and a time range to request, visualize, and analyze the 4D Argo data stored in an optimized database. The fast Argovis database enables users to receive JavaScript Object Notation (JSON) or comma-separated value (CSV) formatted data and Hypertext Markup Language (HTML) figures almost instantly. More advanced users can also query Argovis from other programming environments, such as Python, $\mathrm{R}$, and MATLAB.

Argovis improves on other tools for Argo data access by allowing interactive querying and visualizing of curated profiles on the basis of a 4D spatial-temporal data selection (e.g., retrieving upper-ocean data in the tropical eastern Pacific Ocean during a period of interest). In addition, metadata database queries and additional specialized aggregation queries are under development.

The guiding philosophy of our Argovis development includes visualization with minimal effort, fast delivery, and wide availability for everyone. We referenced the ideas of an interactive and responsive interface for children, making Argovis attractive, self-explainable, and 4D. Argovis is different from the traditional data service technologies, such as file transfer protocol (FTP) and download that passively feed digital data to a user or consume the server's computing resources to generate and push figures (e.g., the NASA Giovanni application). The Argovis system is an active technology instead, displaying data dynamically. The user requests data that are used to render pages on the browser.

For data science experts, our architecture allows for statistical analysis such as optimal interpolation (also known as objective mapping or statistical interpolation), for example, to calculate sea surface height anomalies using Argo temperature and salinity profile data as in Kuragano et al. (2015). Kuragano et al.'s (2015) model depends on fitting a local 3D Gaussian function regulated by small scales, and can represent an anisotropic spatial scale, time scale, and propagation feature of oceanographic variability. The data used to calculate parameters scales for the Gaussian function are gridded $\left(1 / 4^{\circ}\right.$ latitude $\times$ longitude $\times 3$ days $)$, requiring hundreds of thousands of data queries. Argovis's application programming interface (API) querying tool can be very helpful in this instance. In more general terms, Argovis's API makes the process of selecting Argo data flexible and customizable to the needs of each model or researcher. As an interface to a database, the API is designed for such queries, so that data manipulation time is reduced while simultaneously relieving the researcher of the task of optimizing data input or output. The API can also be used for data extraction to create gridded products using other methods, for example, objective mapping in Roemmich and Gilson (2009) or Kuusela and Stein (2018) or spectral optimal gridding in Shen 
et al. (2017). More of this will be covered in the spacetime query section described in section $6 \mathrm{c}$. For users interested, instead, in analyzing profiles locations, dates, DACs, positioning systems, and so on, we have provided a metadata API, whose details and example applications are explained in section $6 \mathrm{~d}$.

In the following sections, we recapitulate the Argo network and its data in section 2 and provide an overview of the web application in section 3. Design practices relevant to Argovis are covered in section 4. Section 5 provides technical details of Argovis, addressing how the database is structured (section 5a), how datasets are added to the database (section 5a), and how the database is connected (section 5b). Section 6 describes how Argovis data are accessed both through the interface for the website (https://argovis.colorado.edu) and through the API. Section 7 presents the summary and conclusions. As supplementary materials to this paper, Argovis tutorials have been developed and are freely available via the tutorial link on the Argovis home page.

Argovis current uniform resource locator (URL) (https:// argovis.colorado.edu) is referenced in this paper. Future changes to the application domain name will be mentioned in the "README" file of the Argovis GitHub repository (https://github.com/tylertucker202/argo-database). Up-todate URLs for Argovis API can be found via the tutorial link on the Argovis home page.

\section{The Argo data network}

Most Argo floats drift at a parking depth of $1000 \mathrm{dbar}$ and make $T / S / P$ measurements every 10 days while rising to the surface (from a profiling depth of $2000 \mathrm{dbar}$ ). A limited number of floats include biogeochemical observations (Claustre et al. 2010; Gruber et al. 2010) and or measure deeper than $2000 \mathrm{dbar}$ (e.g., to depths of 6000 dbar). Once at the surface, Argo floats transmit profile data to national data assembly centers (DACs). These raw measurements undergo automated quality control (QC) tests and adjustments before being sent as netCDF files to the GDACs where they are served on FTP servers. Servers are frequently updated to include additional QC checks by a float's primary investigator. Profile data that undergo automatic QC are known as real-time-mode profiles. Profiles that are inspected by oceanographic experts and passed through an adjustment algorithm (described in Owens and Wong 2009), are known as delayed-mode profiles. The observational cycle of an Argo float produces a data profile. More than two million profiles have been collected through the Argo Program since 2000, and navigating through the large and complex Argo dataset can be challenging. For instance, accessing Argo netCDF files on the GDACs involves parsing through a remote or local archive of files, selecting relevant files, opening the files, and performing analysis. As an alternative, Argovis takes profile data from the GDACs and stores them on a database that is coupled with a front-end interface for charting and data delivery.

\section{Web application overview}

The Argovis main web page, shown in Fig. 1, consists of an interactive map and sidebar with controls, allowing users an intuitive way to navigate through profiles, either by a space-time query or by entering a float's World Meteorological Organization (WMO) number. Argovis home page shows the interactive map, along with the locations of profiles in a 3-day window (whose latest date defaults to "yesterday" and can be changed in the side panel). The interactive map is created using the Leaflet.js library, that is, a tiled web map generator that includes plugin extensions for shapes and images to be displayed on web maps. Clicking and dragging the map pans the view, and zooming can be done by double clicking a spot on the map.

Toggle elements are included in the sidebar, allowing a quick filter for special types of profiles, such as "Biogeochemical" (BGC) or "Deep" floats. Note that these types of floats appear as different colors. BGC floats are displayed as green, and Deep floats are displayed in dark blue, in contrast to the standard yellow profiles. A button on the side panel clears the page from all the profile locations (i.e., all the dots), if needed (e.g., before a selection).

The pop-up window that appears when a dot is clicked contains the latitude and longitude coordinates, the profile's measurement date, and a button that will show the float's previous profile locations (see the pop-up in Fig. 2a). Links to either the profile page (shown in Fig. 3a) or the entire float history (shown in Fig. 2b) are included as well. BGC floats have links to their own page displaying additional parameters. Another way to view a float's trajectory is by typing in the platform's WMO number on the side panel, revealing the platform's history as seen in Fig. 2a.

Different map projections are available by clicking the name of the desired projection near the top of the sidebar. Currently, web Mercator, northern stereographic, and southern stereographic are the only projections available. Only web Mercator uses map tiles; the latter two use a JSON shapefile to show land outlines (see Fig. 2a for a southern stereographic projection map).

In each projection, the map includes a drawing plugin that allows users to create, edit, and delete a polygonal region of interest. When a polygon is created 


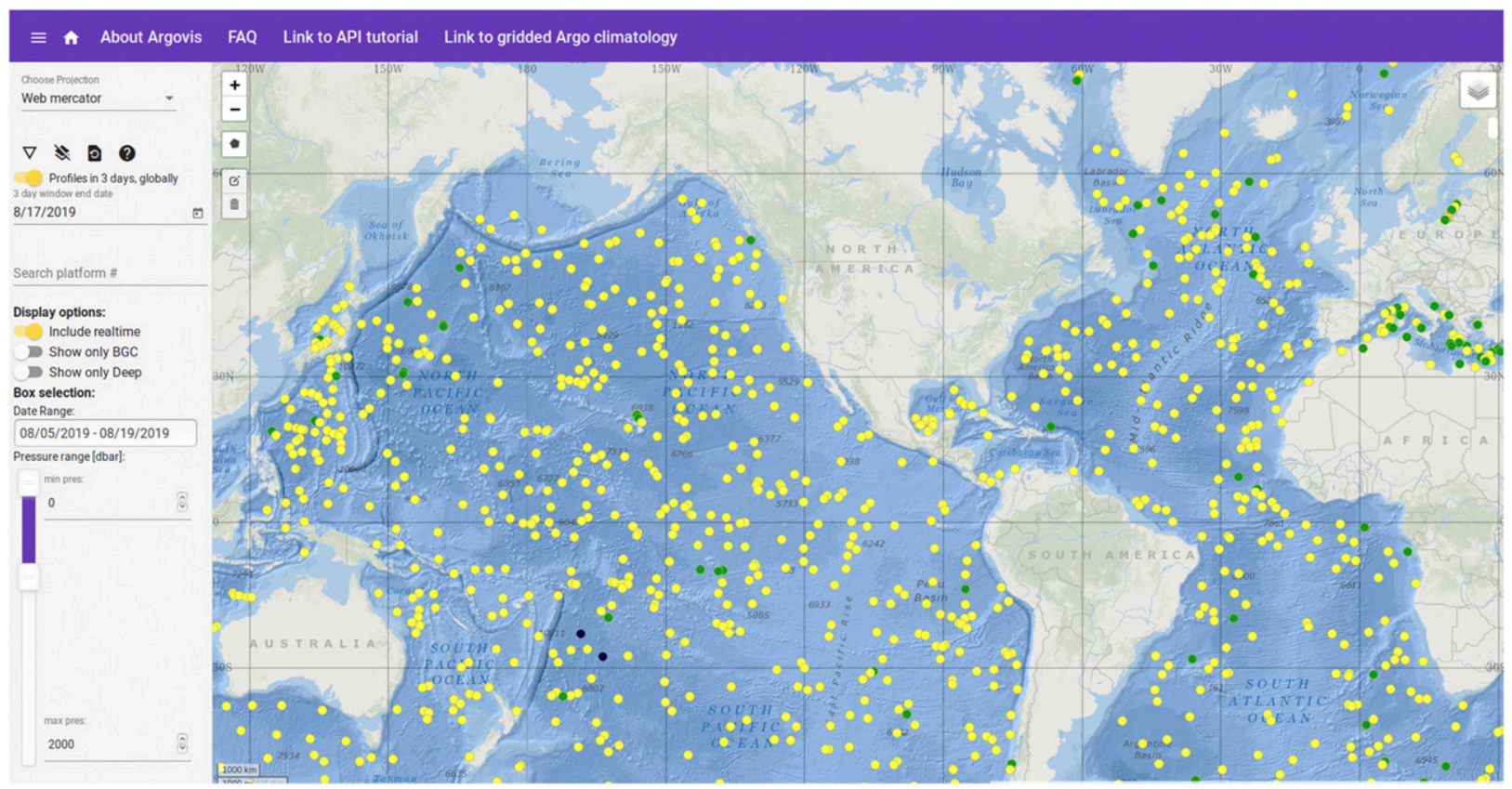

FIG. 1. Users are greeted by a map with the past 3 days of Argo profiles upon visiting the Argovis site (https:// argovis.colorado.edu). The end date of the 3-day window can be changed in the sidebar. Users can query single profiles by either clicking on a yellow/blue/green profile marker or entering a float's WMO number in the platform-number query box. In either case, clicking on the dot opens a pop-up window showing additional information and links to its data visualization page. A shape icon on the map lets the user draw polygons. Upon completion, the map clears all profiles except for the ones that fall within the drawn polygon(s). A pop-up over the shape links to another page with temperature, pressure, and salinity plots for the region of interest. The sidebar includes date and pressure (depth) selection options and buttons to clear the map, filter by profile type, or reset to start.

by drawing it on the map, the application queries the database for profiles falling within the region bounded by the polygon, along with the date and pressure ranges selected on the side panel. If the query is not too large, the application clears the map of profiles and displays profiles that match the temporal-spatial query returned by the database (Fig. 4a). If the query is too large, a message warns the user, with a suggestion to explore API options. The completed shape (e.g., Fig. 4a) will also have a pop-up window with two buttons that open web pages with $T / S / P$ plots for the region and period of interest, with or without the pressure selection (Fig. 4b). The $T / S / P$ plots are interactive and when you click on one profile, a new web page opens showing the $T / S / P$ plots for that single profile (Fig. 3a) and a link to plots for the platform history (Fig. 2b).

Below the $T / S / P$ plots for a drawn section or a platform, there is a table and several buttons for data or table downloading (Fig. 4b). The columns in Fig. 4c are sorted by latitude, longitude, data center, data mode, positioning system, and so on. To access the same region frequently, a user can simply copy the URL of the selection region and reuse it as desired.
The date can be changed in the URL link for a different time period.

\section{Overview of the Argovis web application design}

Modern web framework design and practices pair well with large climate and oceanographic data. Argovis only uses free and open sourced software that are actively developed and maintained by a community of developers.

The tools used to create the Argovis interface as shown in Fig. 1 came from a standard web application design. Web pages are built with HTML and styled with Cascading Style Sheets (CSS). Together these languages display images, buttons, tables, pop-up windows, and so on but do not address event handling such as clicking or scrolling. Logic and event handling are done with JavaScript, which handles the position and zoom level of the map global view. Users import and run HTML, CSS, and JavaScript on their browser as the front end, using their own computing resources.

Complex web applications such as Argovis use frontend frameworks, which are prewritten standardized libraries (i.e., React.js, Vue.js, and Angular). Argovis uses 

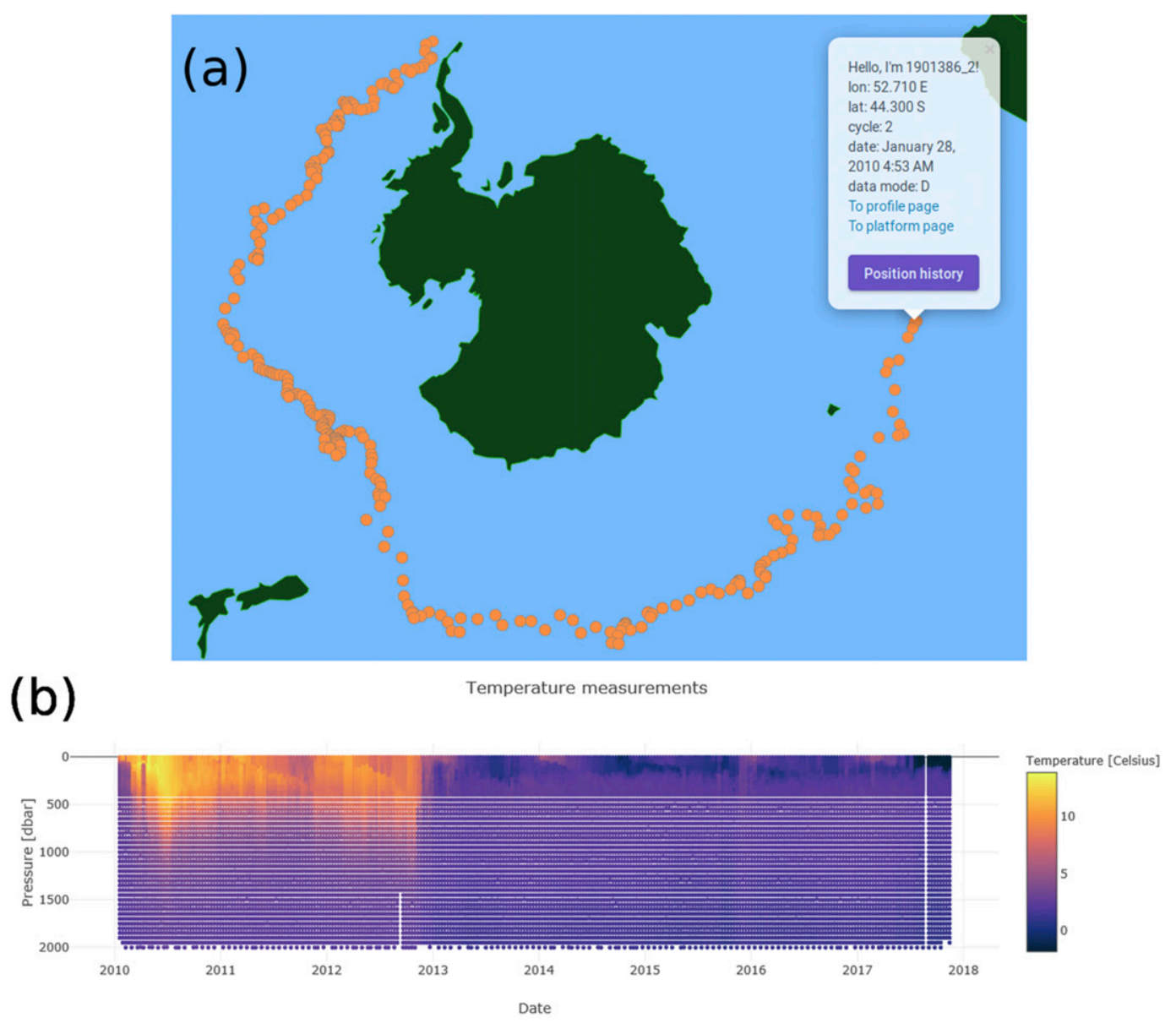

(b)

Salinity measurements

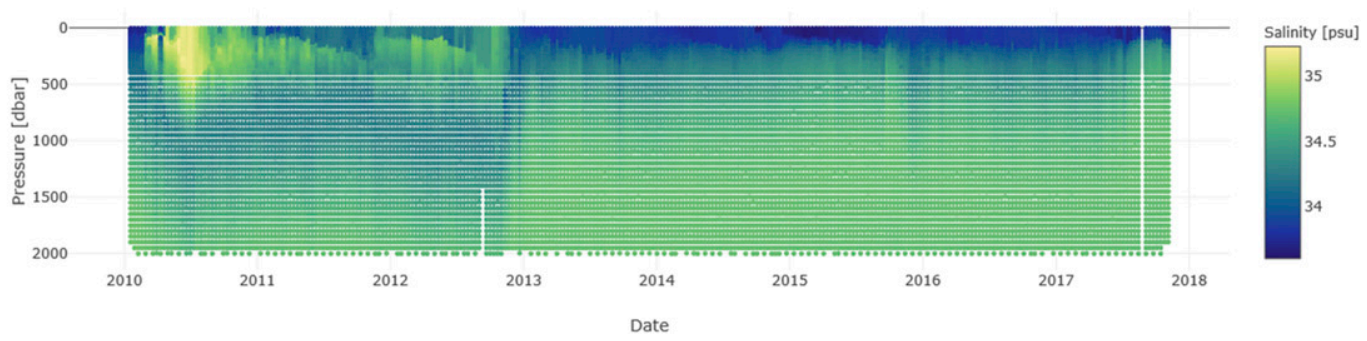

FIG. 2. (a) A trajectory history of float 1901386 on a southern stereographic map projection. Each point represents the float surfacing to transmit its profile data. Clicking on any of the orange profile locations opens a pop-up showing additional information and links to the (b) data visualization page of the float's profiles. The platform page is found online (https://argovis.colorado.edu/catalog/platforms/1901386/page).

Angular libraries as the front end, which are written in TypeScript (i.e., a type-based language that compiles into JavaScript). HTML, CSS, and JavaScript needed to be organized around these libraries to address event handling, requests, and page rendering. When the user makes a selection on Argovis, the front end requests the 4D data, which are delivered in the JSON format via HTTP by Argovis's back end.
Argovis's back end comprises a server, database, and its interface code. Most modern applications such as YouTube or Amazon populate their pages with content stored in databases, allowing users fast and easy access to vast amounts of data such as videos or shopping catalogs. Argovis's profile dataset is stored in a Mongo database (MongoDB). A remote server runs the database and sends HTML, CSS, and JavaScript via HTTP 


\section{(a)}

\section{Profile Id: 5901861_240}

Date: 2015-03-08 13:49

Cycle number. 240

Latitude: $11.700 \mathrm{~N}$

Longitude: $144.326 \mathrm{E}$

Position QC: 1

Dac: aoml

Parameters: PRES, TEMP, PSAL

Positioning system: ARGOS

Platform type: APEX

Data mode: D

\section{Download data as JSON}

Links to other Argovis pages: Link to platform 5901861 page Back to map

External links:

Link to profile data on Argo GDAC Link to Ifremer visualization of this profile Link to platform on JCOMMOPS

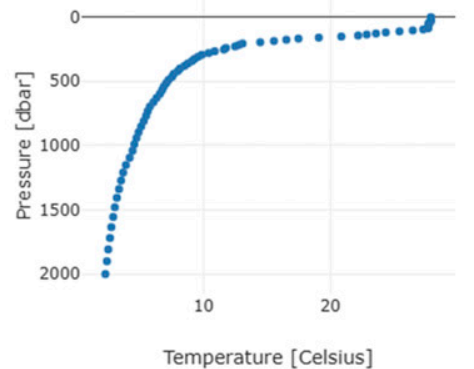

Temperature [Celsius]

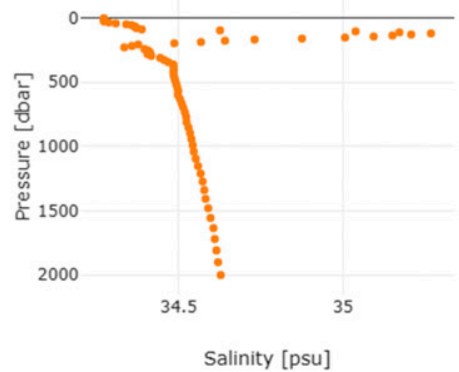

Salinity [psu]

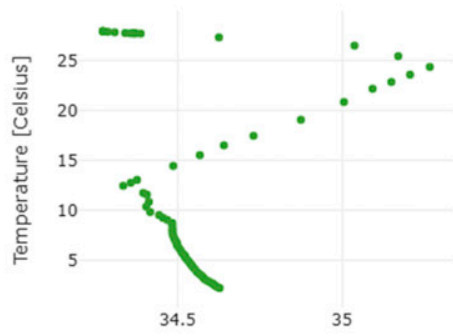

Salinity [psu]

\section{(b)}

date: "2015-03-08T13:49:15.001Z",

date_qc: 1

position_qc: 1 ,

cycle_number: 240 ,

lat: 11.7 ,

lon: 144.326 ,

dac: "aoml",

- geolocation: \{

type: "Point",

- coordinates: [ 144.326,

]

$$
11.7
$$

$$
\text { \}, }
$$

VERTICAL_SAMPLING_SCHEME: "Primary sampling:discrete[discretespotsamplingbySBE-41atreportedpressures]", BASIN: 11 ,

url: "/catalog/profiles/5901861 240",

jcompsplatform: "http://ww.jcommops.org/board/wa/Platform? ref=5901861",

ifremerProfile: "http://ww. ifremer.fr/co-argofloats/cycle? detail=false\&ptfcode=5901861\&cycleNum=240",

- formatted_station_parameters: [

"PRES",

" TEMP",

],

" PSAL"

roundtat: "11.700",

roundLon: "144.326",

strLat: "11.700 N",

strLon: "144.326 E",

date_formatted: "2015-03-08",

id: "5901861 240"

FIG. 3. The (a) profile page and (b) JSON can be respectively accessed by appending or omitting "/page" to the following URL (https:// argovis.colorado.edu/catalog/profiles/5901861_240). 
(a)

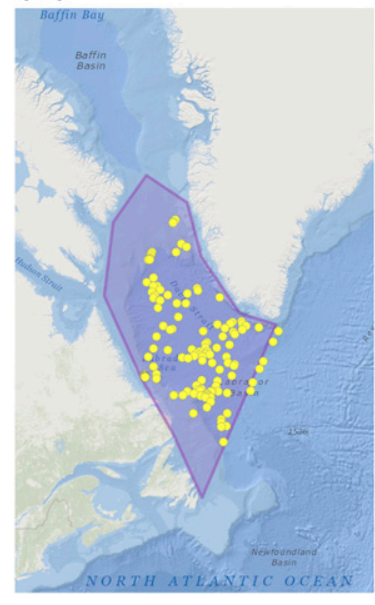

(b)

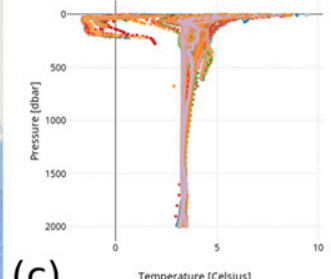

(c)

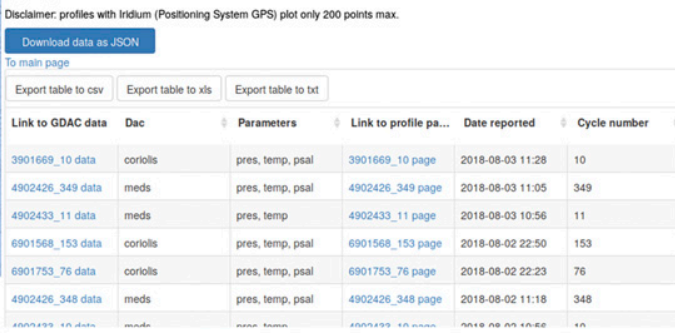

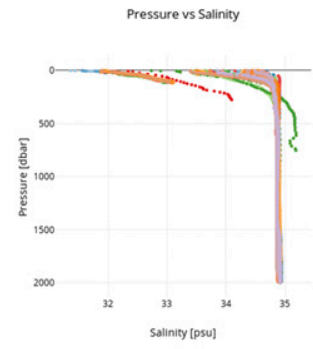

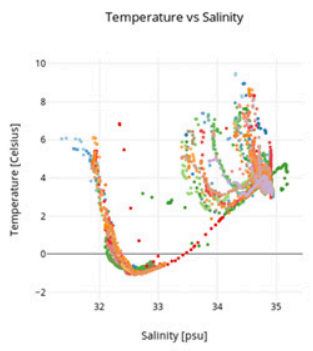

FIG. 4. Upon completion of (a) a shape created by the drawing tool, the map clears all profiles except the ones that fall within the drawn polygon(s). A pop-up over the shape links to another page with (b) temperature, pressure, and salinity plots and (c) a sorted table that can be downloaded.

to users. Code that handles databases and web content has evolved into several specialized libraries known as web frameworks (e.g., Django, Express.js, Flask, and Ruby on Rails). We chose the Express.js framework to handle Argovis page views, URL routing, and database interface.

\section{Argovis development}

\section{a. Selection of MongoDB}

A MongoDB stores Argo profile metadata and their measurements as Binary JSON objects, (i.e., documents). JSON is an easy-to-read, easy-to-write standard consisting of key-value pairs. An example JSON file structure for Argovis is shown as Listing 1 (see Fig. A1 in the appendix). Argo profile data fit within MongoDB's schemaless design. For instance, the "measurements" key in the JSON object generally contains temperature, salinity and pressure, unless the salinity measurement is excluded because of poor-quality observations. A Structured Query Language (SQL) database such as MariaDB or MySQL would require each profile to have a salinity key, even when no salinity is available. MongoDB requires keys for available variables only. This feature is even more relevant for BGC variables (e.g., $\mathrm{O}_{2}$ or $\mathrm{N}_{2}$ ) measured by some of the floats and included in the optional "bgcMeas" key in the JSON object.

In a comparison study of geospatial data between different databases (e.g., MongoDB and SQL-like database based in Apache Hadoop), Hu et al. (2018) show that MongoDB scales well to multiuser query systems for $4 \mathrm{D}$ data. $\mathrm{Hu}$ et al. did not include data insertion time in their analysis. The GDACs update profiles frequently and in bulk, making update time an important criterion for Argovis. We have seen MongoDB updates profiles at a much faster rate while consuming fewer computational resources compared to other options, which ultimately became the deciding factor of database selection.

MongoDB outputs information in JSON format, which is used to render figures and tables on a web page. The JSON file is readily available for downloading, and can be used to render figures in a number of languages using an API described in section 6.

\section{b. Construction of the Argovis database}

\section{1) Architecture}

Query time is reduced substantially when the queried keys of a database are indexed. However, indexing everything adds additional overhead that, if abused, causes a slower performance. The Argovis database has indexed date, platform number, cycle number, $\mathrm{DAC}$, and geolocation, allowing faster query times for these keys.

\section{2) Adding profiles to the database}

The QC'ed Argo data are stored as netCDF files in the GDAC FTP server. We accessed the netCDF Argo data through a mirror of the Ifremer GDAC server at the University of Colorado Boulder using Python scripts that write the netCDF data into the MongoDB. The writing process reformatted the netCDF data into the document schema shown in Listing 1 (see the 
appendix). The Python repository is available online (at https://github.com/tylertucker202/argo-database). The scripts parse netCDF files and add selected metadata and measurements to the database. Of all the profile measurements (e.g., $T / S / P$ ), only values with appropriate QC flags are included. Erroneous data are masked, and missing data are filled in with null values. Using fill values for missing keys goes against MongoDB's schemaless design; however, fill values are included in the measurements key to maintain indexes when plotting data in JavaScript. Array indexes matter when plotting one measurement versus another as shown in Fig. 3a.

The current version of Argovis includes core Argo observations that have QC flag equal to 1 for temperature, pressure, and salinity measurements (i.e., "good" data). Position and date QC flags keep all except QC flags of 3 and 4 (i.e., to exclude profiles with "bad" position). Estimated positions of profiles under sea ice are allowed in Argovis and have a QC flag of 8. Floats trapped under sea ice are not able to transmit their profile data to the DACs immediately, and profiles are delivered when the floats are able to surface; however, position is lost. These profiles have a position QC of 9 and a fill value of $-89^{\circ}, 0^{\circ}$ for latitude and longitude, unless the position is estimated. For Deep Argo profiles, QC flags of 2 for temperature and salinity (i.e., "probably good" data) and 3 for salinity (i.e., "bad" data that are "potentially correctable") are allowed for data below 2000 dbar, per the Argo requirement of flagging all Deep Argo pilot data as 2 or 3 below $2000 \mathrm{dbar}$ [see the link for frequently asked questions (FAQ) on the Argovis home page]. As requested by the BGC community, all QC values are accepted for BGC data (which are a small subset of the dataset), and QC parameters are included for BGC variables. Data are excluded if corresponding pressure values are unknown or bad, as pressure values are needed to generate plots and filter at the front end.

Inside the Argo netCDF data files, measurements can be either nonadjusted (data decoded directly from the raw stream of data reported by the float) or adjusted (data that have been through additional quality control and, if necessary, have been adjusted on the basis of known drifts or offsets). We merge adjusted data in the profile document whenever possible.

\section{3) SYNCHRONIZING DATABASE WITH GDACS}

Argovis reflects near-real-time changes in the GDAC datasets by daily synchronization. Possible changes to the datasets include adding new real-time profiles, replacing real-time-mode profiles with delayed-mode profiles when they become available, or replacing profiles with updated versions.

The "cron" Linux software utility schedules scripts to be run periodically, thereby automating the daily task of updating. Each day, a cron scheduler runs a shell script that updates the local mirror of the Ifremer GDAC using the "rsync" command. The Linux tool rsync copies files that have changed or have been added from the remote GDAC, and it removes files that have been deleted. A list of files that have changed is created and fed to a Python routine that adds or overwrites profile documents to the Argovis database.

\section{Accessing the Argovis database through the API}

There are two ways to access the Argovis database: through the front-end web application (described in section 3) and through the back-end API calls (described in this section). The front end of Argovis for the general public is intuitive, simple and attractive, but can be confining for automated tasks. While our design allows for more visualization features to be added, this is at the expense of additional development cost and maintainability. The back-end API calls allow for fast access to the Argovis database within the users' preferred programming environment and the ability to create additional plots not featured in the front-end web application.

The definition of API is somewhat nebulous; in this context an API is the access to the Argovis database without the need of a browser. RESTfully designed applications (i.e., Representational State Transfercompliant web applications), such as Argovis, allow custom code to retrieve data. This code can be a process running a plotting script, a data archive service, or another website. Hence scientists can access any information in the Argovis database (and even make selections in space and time) using a script in their preferred language by including HTTP requests in their code.

There are four main API calls for Argovis, which can be used to import Argo data in the programming environment of choice, such as Python, R, and MATLAB. Three calls are for accessing profiles: individual profiles, all profiles from one platform, and all profiles in a region, time and pressure range. The fourth call is for accessing metadata associated with the Argovis dataset for the month and year of choice. Table 2 summarizes the input needed for each call and the information that is returned. There is a 16-megabyte size limit for data retrieval per API call, so users are advised to break the calls into chunks of data. Suggestions of how to do this 
TABLE 2. Details for each API type. Boldface font indicates measured variables. Additional information may be found in the output of Argovis API calls: this information is useful for the web application functioning but may not be useful for users.

\begin{tabular}{llll}
\hline \hline \multicolumn{1}{c}{ API call types } & \multicolumn{1}{c}{ Input } & & Output \\
\hline $\begin{array}{l}\text { Profile } \\
\text { Platform }\end{array}$ & Platform no. and cycle no. & lon, lat, position_qc, & Measurements: TEMP, PRES, \\
Region & Platform no. & POSITIONING_SYSTEM, date, & PSAL, and WMO_INST_TYPE \\
& Time range, pressure range, & date_qc, date_added, cycle_number, & \\
& set of lat and lon vertices & platform_number, PLATFORM_ & \\
to define shape & TYPE, DATA_MODE, DATA_ & CENTRE, station_parameters, & PRES_max_for_TEMP, \\
Global metadata & Month and year & station_parameters_in_nc, & PRES_max_for_PSAL, \\
by month & & VERTICAL_SAMPLING_ & PRES_min_for_TEMP, and \\
& & SCHEME, DIRECTION, and & PRES_min_for_PSAL \\
\hline
\end{tabular}

are provided below and on the Argovis tutorial link on the main Argovis page, along with sample Argovis API code.

\section{a. Querying one profile}

When querying one profile, users include the platform number and cycle number in the URL (see example in the caption for Fig. 3). The profile measurements and metadata are returned and can be plotted in a similar manner to Fig. 3a. To search for several profiles, users can create a list of platform and cycle numbers and loop through them to get the desired set of profiles.

\section{b. Querying all profiles from one platform}

When querying all profiles from one platform, users include the platform number in the URL (see example in the caption for Fig. 2). All profile measurements and metadata are returned in the same format as in the query for one profile, except that the output has more dimensions to accommodate the additional profiles. Observations can be plotted in a similar manner to Fig. 2a. If users want the entire dataset, it is recommended to use the space-time query described below and loop through time and small regions rather than loop through all the platform numbers.

\section{c. Querying profiles for a space-time selection}

When querying profiles in a space-time selection, the URL should include the time range, pressure range and latitude and longitude coordinates that define the shape. Profile measurements and metadata are returned in the same format as the query for all profiles from one platform and for instance, bin-averaged values can be plotted easily along with the number of observations in each bin. An example for the Labrador Sea region, whose shape is provided in Fig. 4a, shows such an implementation in Fig. 5. As newer floats with the capability to return more data were deployed starting in 2012, there were more data in each bin (Figs. 5c,d).
In general, measurements are made more frequently in shallower waters to try and capture more rapid temperature changes compared to deeper parts of the water column.

\section{d. Querying profile metadata globally for a certain month and year}

The fourth API call allows users to query metadata associated with the profiles by month and year on a global scale (the URL of an example is https:// argovis.colorado.edu/selection/profiles/10/2010/). For this query, most of the information is the same as for the previous three calls. The only difference is that instead of returning all the temperature, pressure, and salinity measurements, this API call only returns the maximum and minimum pressures available for both temperature and salinity for each profile. This query must be set up in a loop by month and year to get the data over the desired time range.

This query provides easy access to metrics of the Argovis database like the number of profiles available in $1^{\circ} \times 1^{\circ}$ bins as seen in Fig. 6. It is also possible to count the number of profiles that reach certain pressures over time and space (Fig. 7).

The history of data mode can easily be accessed also (Fig. 8). A profile changes from "R" or "A" (real-time mode), to "D" (delayed mode) after being quality controlled by a scientific expert. This should happen starting $12-18$ months after the profile is measured. Following this target, profiles older than 18 months should be in " $\mathrm{D}$ " mode, but this is not yet the case for all profiles (Fig. 8).

The history of the positioning system for profiles is shown in Fig. 9. In the beginning of Argo, only the Argos positioning system was used both to obtain the position of floats and to transmit the data. Newer floats are often deployed using the Iridium satellite system instead, which allows two way communication and faster transmission of larger quantities of data. 


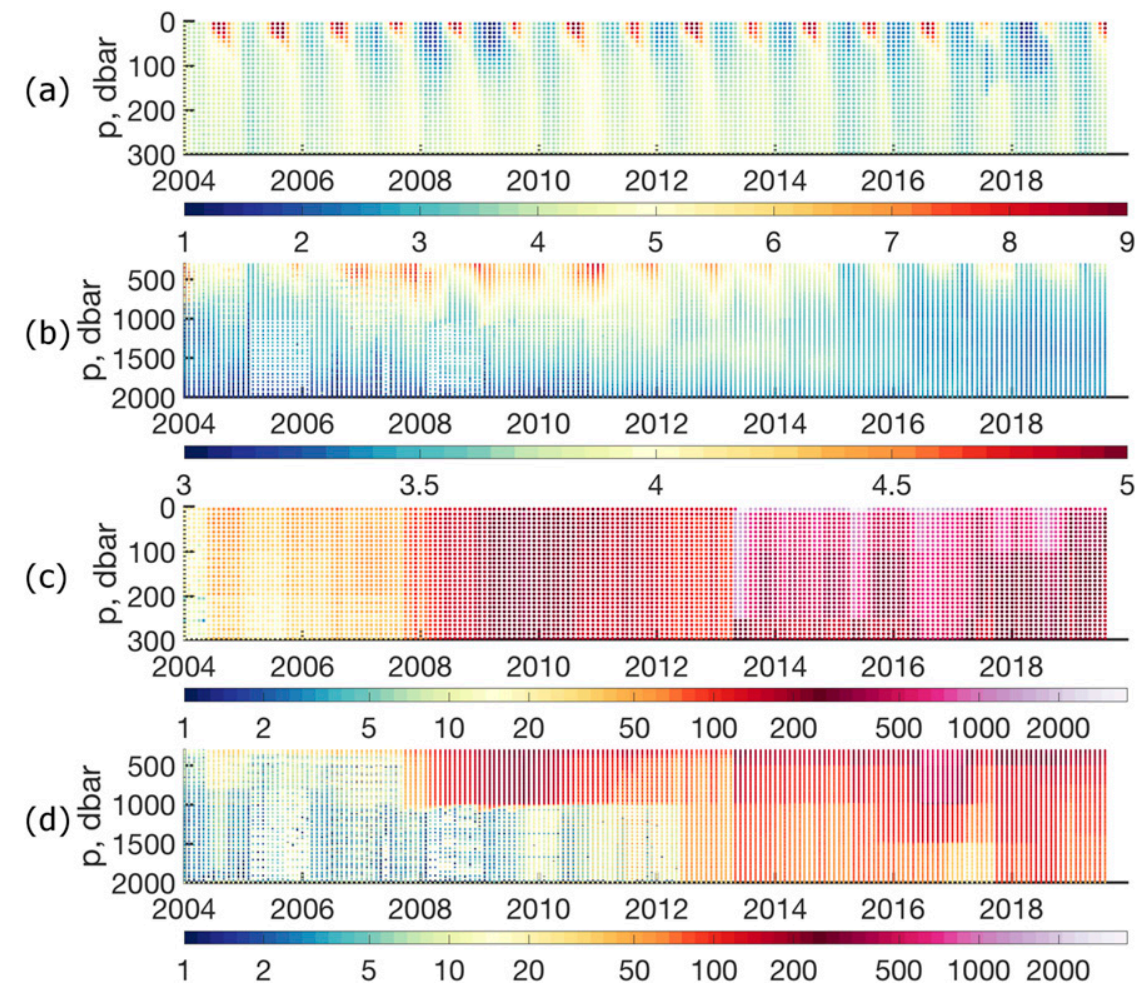

FIG. 5. Labrador Sea (a),(b) bin-averaged temperature in time and (c),(d) number of available observations in each bin. Bins are 10 dbar deep and include data for each month from January 2004 to July 2019.

The quality of the position fixes from Iridium is very low, so GPS is usually used to determine the profile's position. If no GPS fix is available, the lower-quality Iridium fix can be used instead. The number of Iridium floats has been growing over time (Fig. 9), yet given the 3-4-yr lifetime of a float, floats using the original Argos system will still be producing profiles for several years to come. In Fig. 9, the label "Other" indicates profiles for which a typographical error is detected in the transmission system key within the original Argo netCDF file (and hence imported in Argovis).

The stack plot shown in Fig. 10 shows how many profiles are produced by each data center (DAC) in the Argovis database. The American DAC (AOML) has always had the majority of files, but one can track which DACs have grown or reduced in size over time.

Users can also track the types of floats being used in Argo over time as seen in Fig. 11. At the beginning of

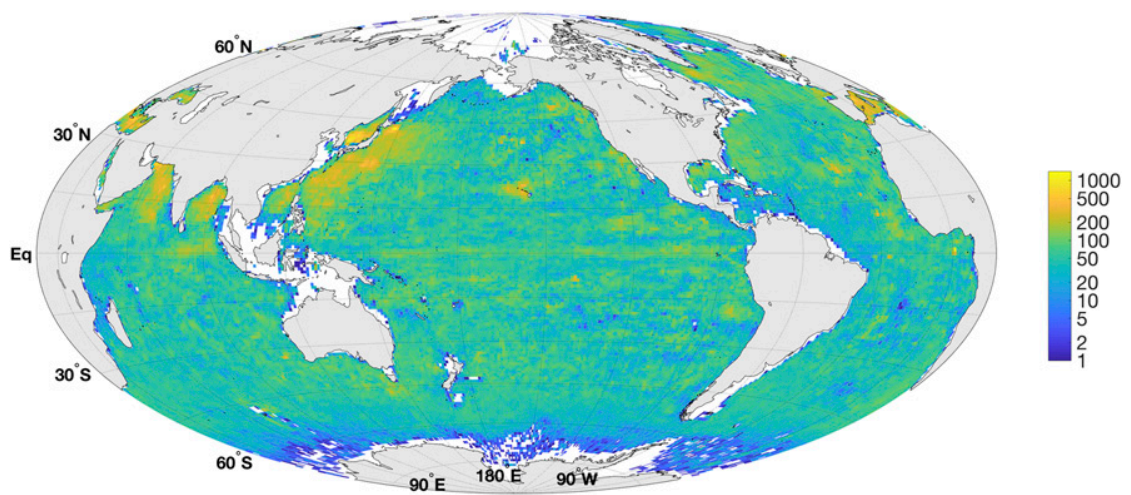

FIG. 6. Density of Argo profiles in $1^{\circ} \times 1^{\circ}$ bins from January 2004 to July 2019. Only profiles with position QC flag of 1 are included. 

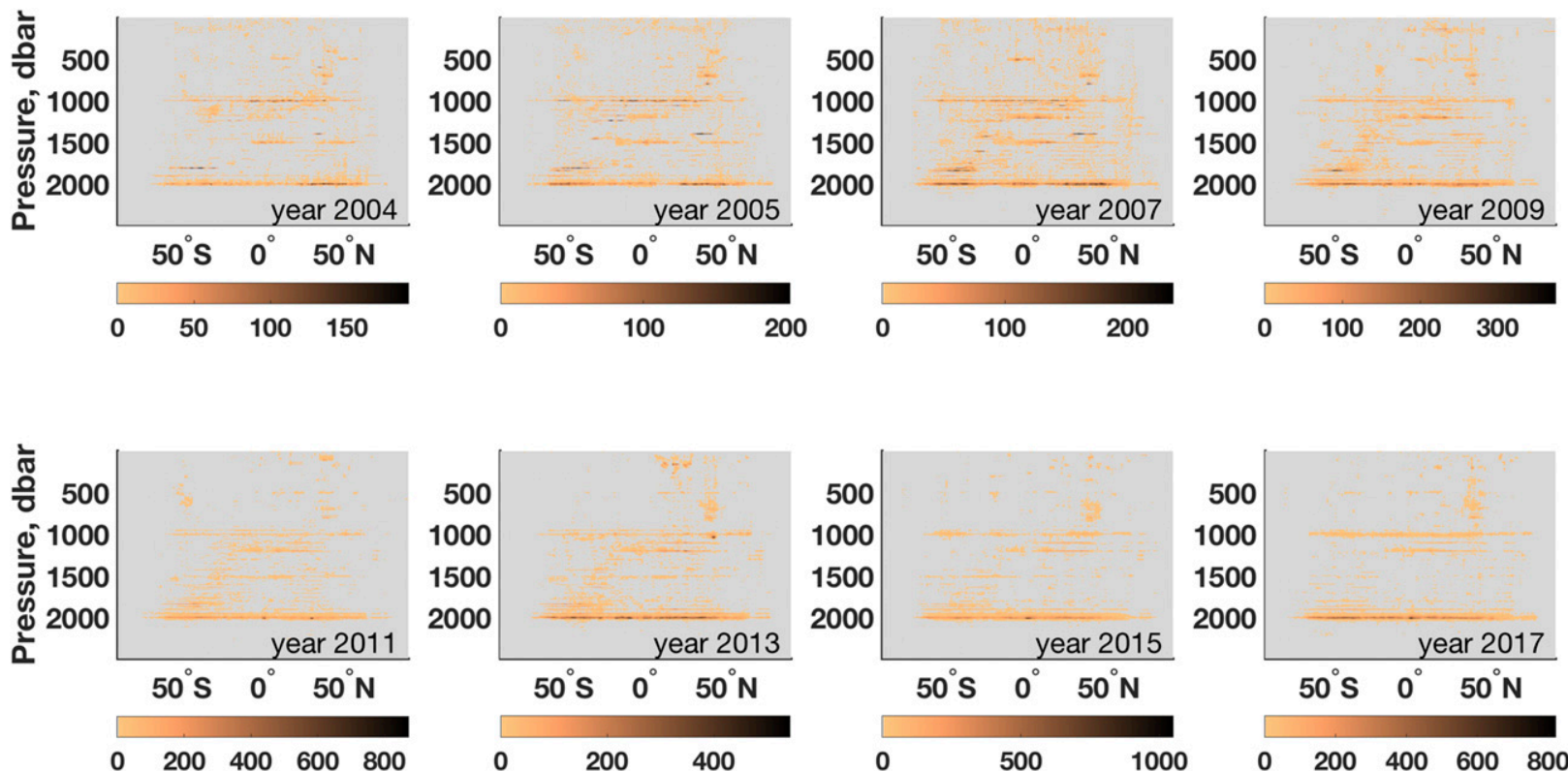

FIG. 7. Number of profiles (colors) with similar maximum profile pressure reached (on the vertical axis) binned by $1^{\circ}$ latitude (on the horizontal axis) from January 2004 to July 2019. Different plots are for different years (see relevant year on the bottom right of each panel). Note that the color bar changes for each plot.

Argo, there were three float types: APEX, SOLO, and PROVOR, but over time more floats have been introduced. Several float types appear around the same time that Iridium communications began because of float redesigns and additions to handle the new hardware. As with the DACs, it is interesting to see which float types gain or lose market share within the Argo Program.

One may also wish to see how deep Argo profiles propagate around the world in a year. Figure 7 shows the number of profiles with a similar maximum profile pressure reached binned by $1^{\circ}$ latitude over time.

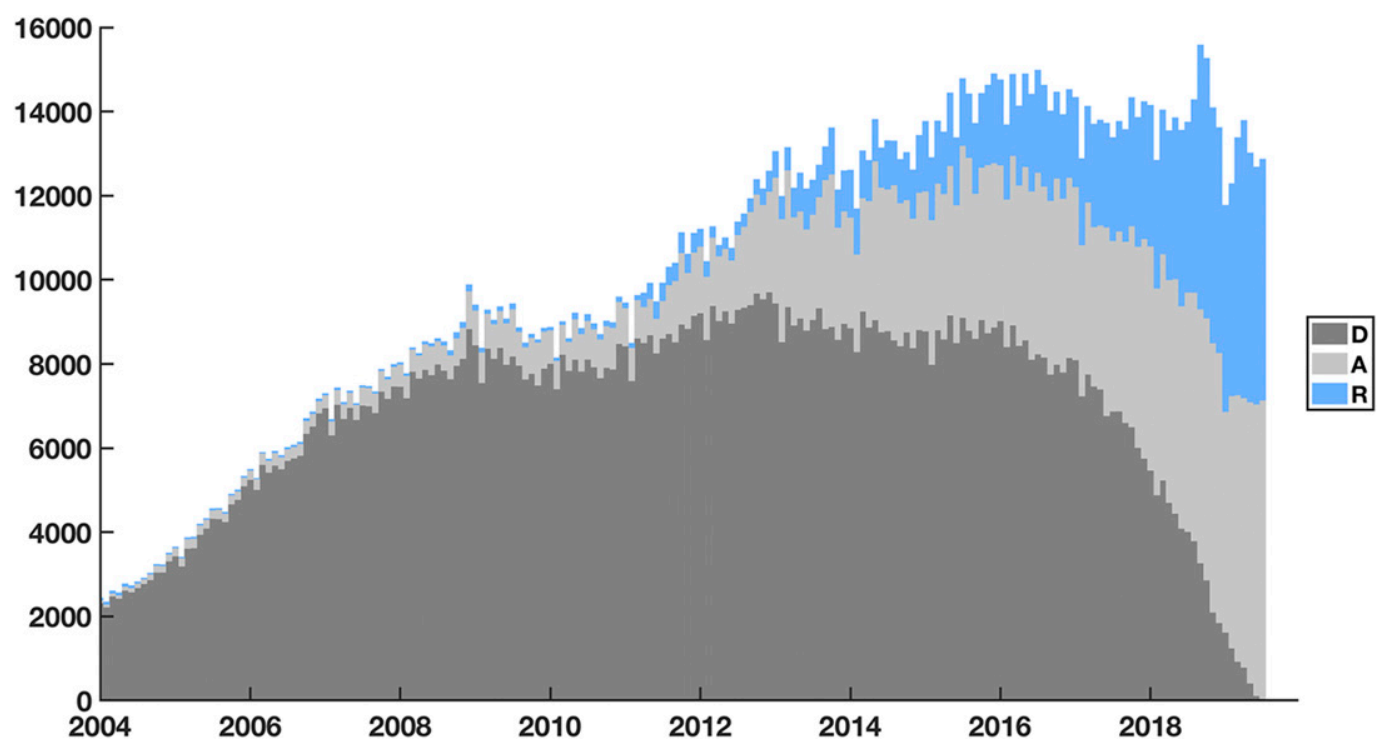

FIG. 8. Data-mode time series from January 2004 to July 2019 for Argo profiles currently in Argovis. Floats in " $D$ " mode have been delayed-mode quality controlled by a scientific expert. Floats in "A" mode are real-timemode profiles that have been adjusted to apply a known pressure or salinity offset. Floats in "R" mode are realtime-mode profiles without any adjustments made. 


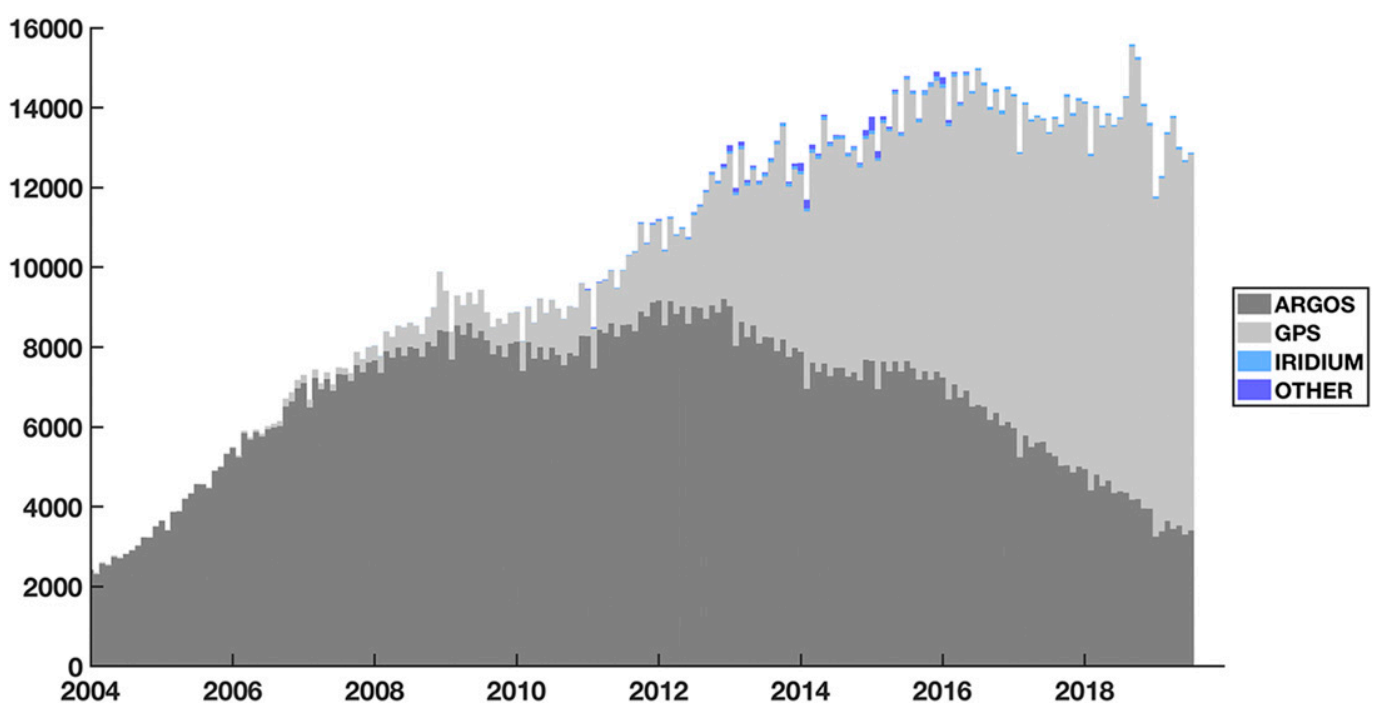

FIG. 9. History of positioning system used for Argo profiles from January 2004 to July 2019. Older floats used the Argos positioning systems, whereas newer floats often use GPS to obtain a position and the Iridium satellite system to send larger amounts of data upon surfacing. If a GPS fix is not available, sometimes the Iridium satellite can give a position fix.

The majority of Argo floats are programmed to profile from 2000 dbar. A darker band near 2000 dbar (in Fig. 7) means that a large number of profiles reach this depth. There is still a spread of profiles from shallower depths, especially earlier in the Argo Program when most float types had difficulty reaching $2000 \mathrm{dbar}$ in regions of high density stratification because they could not adjust their buoyancy properly. Newer float models, starting in 2014 , do not have these problems.

\section{Summary and conclusions}

Argovis is a modern web tool for cutting-edge oceanographic applications of data visualization and

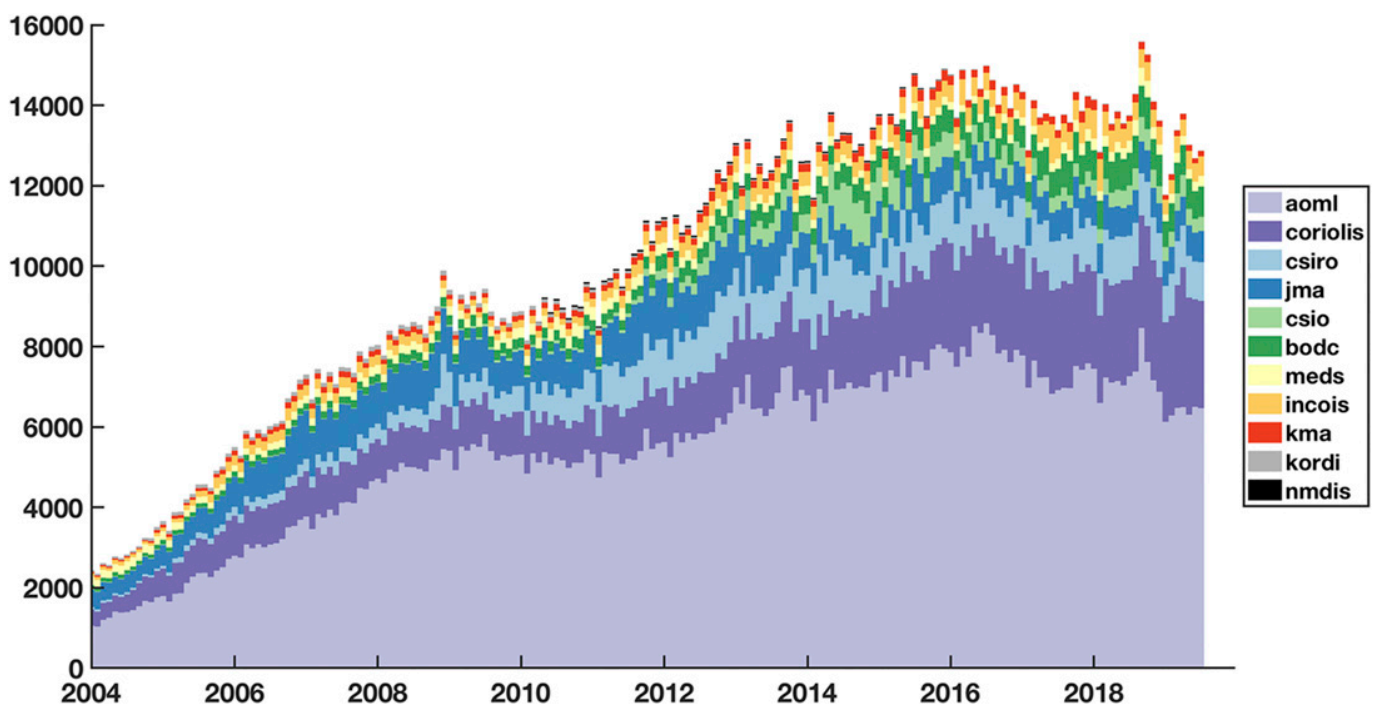

FIG. 10. Argo profiles have been created by DACs in different countries around the world from January 2004 to July 2019. This plot shows the distribution of profile ownership over time by the various DACs in Argo. AOML is in the United States. Coriolis is in France. CSIRO is in Australia. JMA is in Japan. CSIO is in China. BODC is in the United Kingdom. MEDS is in Canada. INCOIS is in India. KMA and KORDI are both in Korea, and NMDIS is in China. 


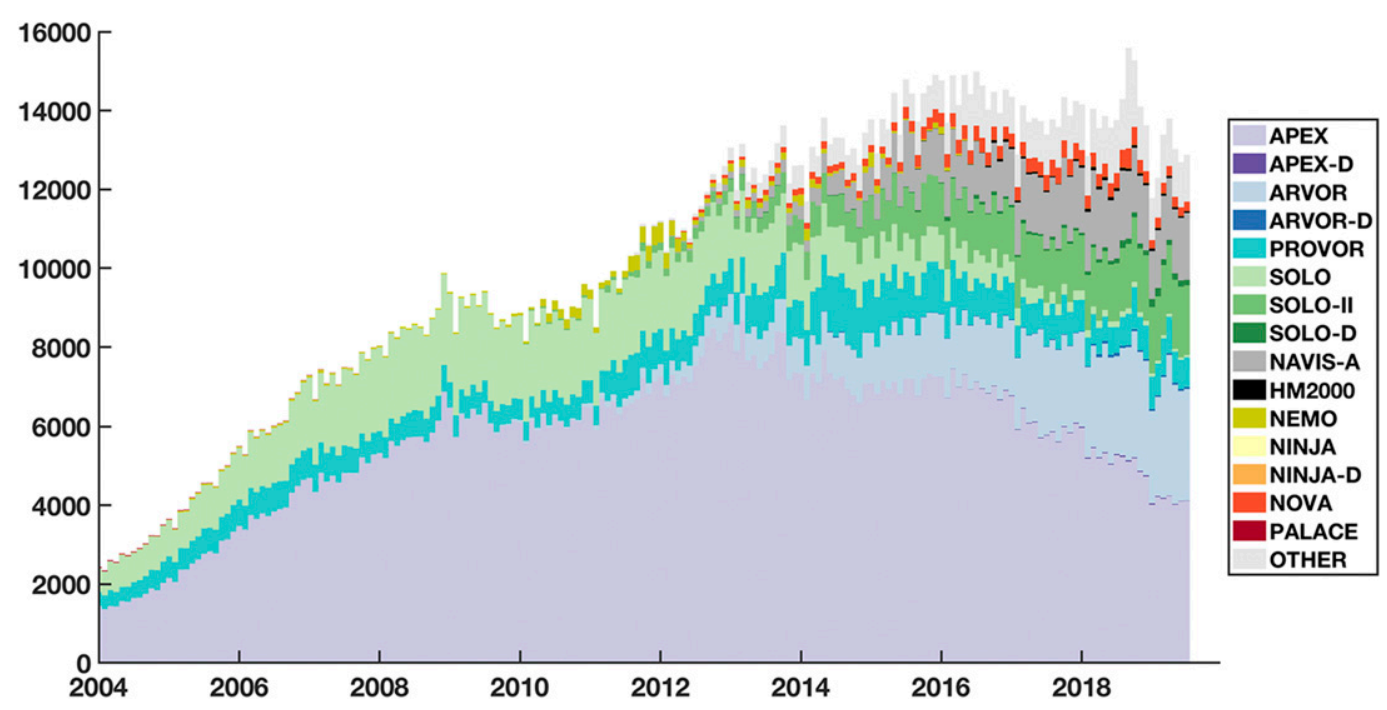

FIG. 11. History of float types used in Argo. There were fewer float types at the beginning of Argo-mainly APEX, SOLO, and PROVOR floats. Newer float types include the SOLO-II (a redesign of the SOLO using Iridium communications), the ARVOR (a compact redesign of the PROVOR), and the NAVIS-A, which is most similar to the APEX float. The float types with "-D" are Deep Argo floats that go to 4000 or 6000 dbar. The other float types have generally not been used in large numbers across several programs contributing to Argo.

delivery, featuring an ease-of-use interface and flexible ways of providing a curated set of Argo observations.

Argovis requires little to no training other than the basic "point and click" computer proficiency. Support from the general public and the broader scientific community is helpful for scientific programs such as Argo to thrive. Open web applications such as Argovis allow nonscientists to visualize government funding at work to bolster their support for such funding. Public kiosks at museums or ocean science centers around the world can display Argovis. Secondary-education schools may use Argovis in their science education, which may even motivate some students to "adopt" a float by following the float's data and broadcasting the float's records.

Improvements to Argovis's ergonomic and convenient design encourages use also by scientifically inclined users, ultimately favoring discoveries by increasing the number of studies that analyze Argo observations. Temperature and salinity measurements are needed not only by physical oceanographers, but also by biologists, engineers, climate scientists, and even the general public. Such a diverse group of users undoubtedly have preferred computing tools and programming languages. The API for Argovis tailors to the needs of the Argo community. The API calls described in section 6 and on the tutorial page of Argovis (via the tutorial link on the Argovis home page) show just how simple hooking up to Argovis can be. Float data can be extracted in JSON format and parsed into a tabular format like CSV or ASCII in a few lines of code. It is our thought that Argo scientists will write (and share) the four functions described in this manuscript, in a language of their own preference such as $\mathrm{R}$ and Julia. Also, automation through the API ensures scientists can spend more time on the nontrivial statistical analysis and mathematical modeling rather than obtaining data through a browser or FTP, and then decoding and selecting the data with appropriate QC flags.

Existing and continuing collaboration with the community will further add to the value of Argovis, improving the work flow for scientists already familiar with Argo observations and serving those not yet using these data. In the future, Argovis will be extended to include other datasets, leveraging the web application's architecture toward a comprehensive data display and delivery system for geosciences. Also, visualization tools for gridded fields are under development, which will replace the static global images of different Argo products currently shown on the gridded climatology section of the website. Included in this development is a tool that displays float trajectory probability fields. Its most recent version is available online (at https:// argovis.colorado.edu/ng/covar).

Acknowledgments. Argovis is hosted on a server of the Department of Atmospheric and Oceanic Sciences (ATOC) at the University of Colorado Boulder. Special thanks are given to Guilherme P. Castelao and Lisa Lehmann, who provided assistance in the Argo data access. Thanks are also given to Dean Roemmich and Lynne Talley for providing feedback, enthusiasm, and 


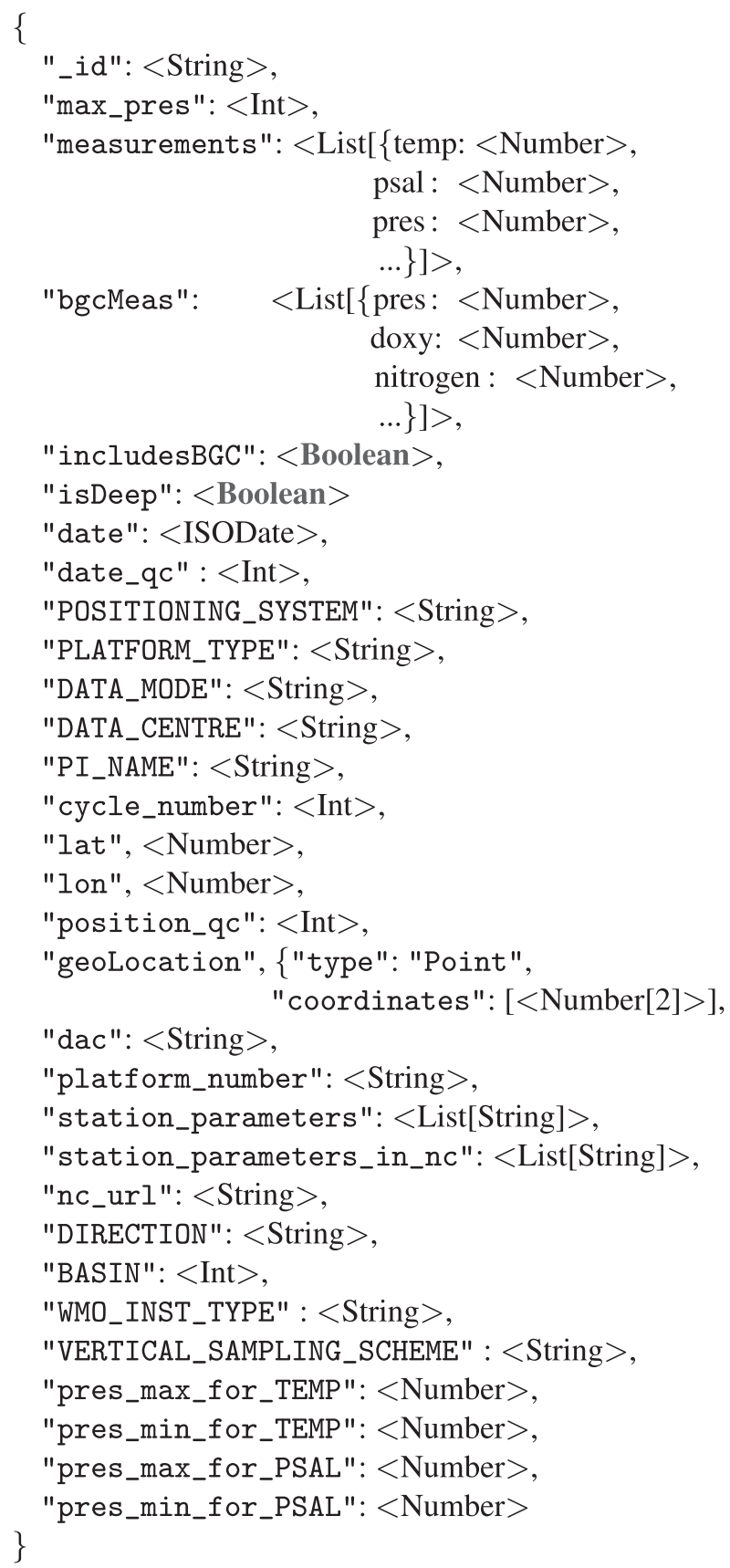

FIG. A1. Listing 1: JSON schema of MongoDB profile.

encouragement. For his advice and guidance on implementing the Angular web framework, we thank Julian Pierret. This study was supported by the National Oceanic and Atmospheric Administration Cooperative Science Center for Earth System Sciences and Remote Sensing Technologies (NOAA CREST) under the Cooperative Agreement Grant NA16SEC4810008, Giglio's research funds provided by University of Colorado Boulder, NSF EarthCube (Award 1928305), the U.S. NOAA
Cooperative Institute for Climate Science (Award 13342-Z7812001), the U.S. Argo Program through NOAA Grant NA15OAR4320071 (CIMEC), and the SOCCOM Project through Grant NSF PLR-1425989. Tyler Tucker thanks the City College of New York, NOAA CREST program and NOAA Office of Education, Educational Partnership Program for full fellowship support at San Diego State University. Also he thanks STATMOS (the Research Network for Statistical Methods for Atmospheric and Oceanic Sciences) and the U.S. National Science Foundation for travel support. The statements contained within this paper are not the opinions of the funding agency or the U.S. government but reflect only those of the authors. Argo data are collected and made freely available by the International Argo Program and the national programs that contribute to it (http://www.argo.ucsd.edu; http:// argo.jcommops.org). The Argo dataset (http://doi.org/ 10.17882/42182) used in this paper was downloaded from the Ifremer GDAC (ftp://ftp.ifremer.fr/ifremer/argo).

\section{APPENDIX}

\section{JSON Schema of MongoDB Profile}

Listing 1 (Fig. A1) shows an example JSON file structure for Argovis.

\section{REFERENCES}

Argo France, 2019: Argo active network map. Argo France, http:// www.argo-france.fr/en/welcome/.

Claustre, H., and Coauthors, 2010: Bio-optical profiling floats as new observational tools for biogeochemical and ecosystem studies: Potential synergies with ocean color remote sensing. Proc. OceanObs'09: Sustained Ocean Observations and Information for Society, Venice, Italy, ESA, https://doi.org/ 10.5270/OceanObs09.cwp.17.

Gruber, N., and Coauthors, 2010: Adding oxygen to Argo: Developing a global in situ observatory for ocean deoxygenation and biogeochemistry. Proc. OceanObs'09: Sustained Ocean Observations and Information for Society, Venice, Italy, ESA, https://doi.org/10.5270/OceanObs09.cwp.39.

$\mathrm{Hu}, \mathrm{F}$., and Coauthors, 2018: Evaluating the open source data containers for handling big geospatial raster data. ISPRS Int. J. Geoinf., 7, 144, https://doi.org/10.3390/ijgi7040144.

JCOMMOPS, 2019: The WMO-IOC Joint Technical Commission for Oceanography and Marine Meteorology In Situ Observations Programme Support Centre. Argo France, http://argo. jcommops.org.

Kuragano, T., Y. Fujii, and M. Kamachi, 2015: Evaluation of the Argo network using statistical space-time scales derived from satellite altimetry data. J. Geophys. Res. Oceans, 120, 45344551, https://doi.org/10.1002/2015JC010730.

Kuusela, M., and M. L. Stein, 2018: Locally stationary spatiotemporal interpolation of Argo profiling float data. Proc. Roy. Soc., 474A, 20180400, https://doi.org/10.1098/RSPA.2018.0400. 
Owens, W. B., and A. P. Wong, 2009: An improved calibration method for the drift of the conductivity sensor on autonomous CTD profiling floats by $\theta-S$ climatology. Deep-Sea Res. I, 56, 450-457, https://doi.org/10.1016/j.dsr.2008.09.008.

Pierret, J., and S. S. P. Shen, 2017: 4D visual delivery of big climate data: A fast web database application system. $A d v$. Data Sci. Adapt. Anal., 9, 1750006, https://doi.org/10.1142/ S2424922X17500061.

Roemmich, D., and J. Gilson, 2009: The 2004-2008 mean and annual cycle of temperature, salinity, and steric height in the global ocean from the Argo Program. Prog. Oceanogr., 82, 81-100, https://doi.org/10.1016/j.pocean.2009.03.004.
- , and Coauthors, 2009: The Argo Program: Observing the global ocean with profiling floats. Oceanography, 22 (2), 3443, https://doi.org/10.5670/oceanog.2009.36.

Shen, S. S. P., G. P. Behm, Y. T. Song, and T. Qu, 2017: A dynamically consistent reconstruction of ocean temperature. J. Atmos. Oceanic Technol., 34, 1061-1082, https://doi.org/ 10.1175/JTECH-D-16-0133.1.

Smith, C. A., G. P. Compo, and D. K. Hooper, 2014: Web-Based Reanalysis Intercomparison Tools (WRIT) for analysis and comparison of reanalyses and other datasets. Bull. Amer. Meteor. Soc., 95, 1671-1678, https://doi.org/10.1175/BAMS-D13-00192.1. 